\title{
Correlates of anemia among women of reproductive age in Ethiopia: Evidence from Ethiopian DHS 2005
}

\author{
Samson Gebremedhin ${ }^{1}$, Fikre Enquselassie ${ }^{2}$
}

\begin{abstract}
Background: Globally, $41.8 \%$ of pregnant women and $30.2 \%$ of non-pregnant women are anemic. Previous studies which attempted to identify determinants of anemia among women of reproductive age reported conflicting findings. Objective: To assess the correlates of anemia among women of reproductive age in Ethiopia.

Methods: A quantitative cross-sectional study carried out based on the secondary data of the Ethiopia Demographic Health Survey (EDHS) 2005. Data of a total of 5963 women of reproductive age were included in the analysis. Data were mainly analyzed using ANOVA and binary logistic regression.

Result: The prevalence of anemia was $27.4 \%$ (95\% CI: 26.3-28.5\%). Rural residence, poor educational and economic status, 30-39 years of age and high parity were key factors predisposing women to anemia. Lactating women and those who gave birth in the month of the interview had $1.3(p=0.000)$ and $2.2(p=0.012)$ times higher risk than their counterparts. Those not using contraceptive were 1.4 times $(p=0.02)$ more likely to develop anemia than current contraceptive users. The average Dietary Diversity Score (DDS) was only 4.01, and not more than $15 \%$ of the respondents consumed iron rich foods in the preceding day of the survey. Respondents with low DDS and those who did not consume iron rich foods in the reference period had significantly higher risk of anemia with odds ratio of 1.3 ( $p$ $=0.01)$ and $1.3(p=0.002)$, respectively. Utilizing maternity services, taking iron and vitamin A supplement during pregnancy and postpartum period, respectively, didn't have a significant effect in reducing the burden of anemia.

Recommendation: Family planning, economic and educational empowerment of women have affirmative inputs in combating anemia. A combination of nutrition, educational and livelihood promotion strategies should be instated to enhance dietary diversity. Maternal nutrition interventions should be integrated in a stronger manner into maternity services. [Ethiop. J. Health Dev. 2011;25(1):22-30]
\end{abstract}

\section{Introduction}

Anemia is a disorder which is principally characterized by a decline in the concentration of circulating erythrocytes or hemoglobin in the blood and a concomitant impairment of oxygen transportation (1). Common etiological classification identifies nutritional, marrow disease and hemolytic anemia types. Nutritional anemia is by far the most common type worldwide and mainly includes iron, folate and vitamin $\mathrm{B}_{12}$ deficiencies (2).

Anemia is a global public health problem affecting both developing and developed countries (3) and occur at all stages of the life cycle, but is more prominent in pregnant women, young children and other women of reproductive age (1-3). Consequences of anemia include increased risk of adverse pregnancy outcomes, maternal mortality, child mortality, impaired neuro-cognitive and physical development of children and reduced work capacity $(4,5)$.

According to WHO, based on the studies conducted from 1993 to 2005, the estimated global prevalence of anemia was $24.8 \%$, and it affected 1.62 billion people worldwide. Estimated prevalence was $41.8 \%$ in pregnant women and $30.2 \%$ in non-pregnant women. In numbers, 56 million pregnant women and 468 million nonpregnant women were affected (3). Furthermore, many more are likely to have not apparent iron deficiency (2). In 2002, iron deficiency anemia was considered to be among the most important contributing factors to the global burden of diseases, attributed to $3.1 \%$ of the total Disability Adjusted Life Years (DALY) lost in the developing world (6).

Studies conducted over the years witnessed the public health significance of anemia in Ethiopia. A study in 1999 by Haidar et al (7) reported that $18.4 \%$ of pregnant and lactating women in rural Ethiopia were anemic. In 2006, Micronutrient Initiative (MI) Ethiopia estimated $27.0 \%$ and $30.6 \%$ prevalence of anemia among women of reproductive age and pregnant women, respectively (8). The Ethiopian DHS 2005 documented that 27\% of Ethiopian women were anemic (9). Recent nationwide surveys reported $29.4 \%$ and $30.5 \%$ prevalence in women of reproductive age $(10,11)$.

A handful of previous studies tried to identify potential socio-cultural, economic, demographic, nutritional, reproductive and other potential risk factors of anemia in women of reproductive age (12-18). However, in addition to being small scale, these studies reported conflicting and divergent conclusions. Hence, this study was done to identify correlates of anemia among women of reproductive age in Ethiopia using large scale EDHS

${ }^{1}$ Hawassa University, College of Agriculture, Tel. +251916822814, E-mail samsongmgs@yahoo.com, P.O. Box

12485, Addis Ababa, Ethiopia;

${ }^{2}$ School of Public Health, Addis Ababa University. 
2005 data.

\section{Methods}

Study Setting: Ethiopia is situated in the horn of Africa. It is a country with great climatic, geographic and cultural diversity. According to the 2008 estimate, it has a population of $78,254,090$ of which $84 \%$ live in rural areas. More than one fifth (23.3\%) of the population are women in the reproductive age (19). The country is characterized by rapid population growth with an annual rate of $2.6 \%$ and a total fertility rate of 5.4, and low GDP per capita of $173 \operatorname{USD}(9,19,20)$.

In Ethiopia, maternal health indicators are seriously low. The maternal mortality ratio of 673 per 100,000 live births is among the highest in the world with only $5 \%$ of Ethiopian mothers delivering in health institutions and, ANC and PNC coverage are $27.6 \%$ and $6.1 \%$, respectively. Likewise, the contraceptive prevalence rate is $14 \%$. More than a quarter of women of reproductive age have chronic energy deficiency (9).

Study Design: This is a quantitative, cross-sectional comparative study based on secondary data of Ethiopia DHS 2005.

Data Extraction: The Ethiopian DHS 2005 data were downloaded from Measure DHS website in SPSS format. Further data cleaning was done by the investigators. Data on a total of 5963 women of reproductive age were included in the analysis. Information on a wide-range of potential independent variables (socio-demographic, economic, dietary intake, nutritional status, micronutrient supplementation history, breastfeeding history, maternity services utilization, family planning use, fertility history etc) were extracted accordingly.

Sampling Method of the Ethiopian DHS 2005: The Ethiopian DHS 2005 was designed to provide representative estimates of health and demographic indicators at national level and across 11 geographic areas (9 regions and 2 city administrations) of the country. Stratified and cluster sampling was used to identify study subjects. The stratification was made based on de-facto place of residence (urban/rural). The samples were selected in two stages. In the first stage, 540 clusters (145 urban and 395 rural) were selected from the list of enumeration areas of the 1994 population and housing census. Then a representative sample of approximately 14,500 households was selected for the main study. For the anemia study, among women of reproductive age, one in two households selected for the main study were identified, and representative samples of approximately 6000 women of reproductive age were included (9).

Data Collection Method of the Ethiopian DHS 2005: The survey employed standard DHS questionnaire which is used across similar surveys in the world. However, the tool was adapted to the socio-cultural setting of Ethiopia through experts' review. The questionnaires were finalized in English and translated into the three main local languages: Amharic, Oromiffa and Tigrigna. Prior to data collection, the questionnaires were pretested. The data were collected using experienced and qualified data collectors in the presence of intensive supervision. Dietary intake was assessed using 24 hours dietary recall method. Blood hemoglobin level was determined using the HemoCue portable meter. Hemoglobin level was adjusted for altitude of respective clusters using the formula recommended by Centers for Disease Control and Prevention (CDC) (9).

Data Analysis: The data analysis was done by the principal investigators using SPSS for windows version 16.0. Frequencies, percentage, mean and standard deviation were used for the descriptive analysis. Weighted percentage was used to compute national anemia prevalence. Independent sample t-test and Oneway Analysis of Variance (ANOVA) with Bonferroni post hoc test were applied to compare mean blood hemoglobin level across different categories of the independent variables. Binary logistic regression was employed to control potential confounders and to explore association between dependent variable (anemia status) and a wide range of the aforementioned independent variables. Variables were entered into the model using the "enter" method. During the analysis, the fitness and statistical assumptions of the logistic model were checked to be satisfied. Hosmer-Lemeshow statistic was used to assess the fitness of the model. P value of 0.05 was taken as the level of significance.

The Dietary Diversity Score (DDS) was computed from a single 24 hours dietary recall data according to the recommendation of Food and Nutrition Technical Assistance Project Scale Version 2 (21). Food items and liquids consumed in the preceding day and night of the survey were categorized into 12 groups (cereals, root/tubers, pulses/legumes/nuts, vegetables, fruits, meat/poultry, eggs, fish/seafood, sugar/honey, milk/milk products, oil/fats, and miscellaneous) (21). Consuming a food item from any of the aforementioned groups earned a score of 1 for that specific category. If not, a score of 0 was given. Accordingly a DDS of 12 points was developed (21). Then the DDS was categorized into three categories, namely low (DDS $\leq 3$ ), medium (DDS $=4$ or 5 ) and high (DDS $\geq 6$ ) groups. The cutoff points were selected as they had been frequently applied in similar previous studies (22-24).

Wealth index, composite indicator of a cumulative living standard, is calculated based on ownership of selected assets, such as televisions and bicycles, materials used for housing construction, and types of water source and sanitation facilities. The five quintiles (lowest, second, middle, fourth, and highest) were generated using Principal Components Analysis (PCA) statistical technique. This study used pre-computed wealth index values. 
Ethical Issues: The data were downloaded and used after the purpose of the analysis was communicated and permission was taken from Measure DHS Organization. The original data were collected in confirmation with international and national ethical guidelines.

\section{Operational definitions}

The following operational definitions were applied in this study;

- Severe anemia: Women with $<7.0 \mathrm{~g} / \mathrm{dl}$ of blood hemoglobin level.

- Moderate anemia: Women with 7.0-9.9 g/dl of blood hemoglobin level.

- Mild anemia: Pregnant women with 10.0-10.9 g/dl blood hemoglobin level and non-pregnant women with 10.0-11.9 g/dl blood hemoglobin level.

- Low Body Mass Index (BMI): Non-pregnant women with BMI less than $18.5 \mathrm{~kg} / \mathrm{m}^{2}$.
- Normal BMI: Non-pregnant women with BMI of $18.5-25.0 \mathrm{~kg} / \mathrm{m}^{2}$.

- Low Dietary Diversity: DDS less than or equal to three.

- Medium Dietary Diversity: DDS of four or five.

- High Dietary Diversity: DDS greater than or equal to six.

\section{Results}

\section{Socio-demographic Information}

The data on 5963 women in the reproductive age were included in the analysis. The mean $( \pm \mathrm{sd})$ age of the respondents was 28 years ( \pm 9.4 years). More than two third $(72.6 \%)$ of them were from rural areas. Most $(62.6 \%)$ were married or living together with partners. Concerning educational status, more than half $(61.0 \%)$ had no formal education. The socio-demographic information of the participants is summarized in Table 1.

Table 1: Socio-demographic information of the respondents, Ethiopia, 2005

\begin{tabular}{|c|c|c|}
\hline Variables & Frequency $(n=5963)$ & Percentage \\
\hline \multicolumn{3}{|l|}{ Age in years } \\
\hline $15-19$ & 1397 & 23.4 \\
\hline $20-24$ & 1089 & 18.3 \\
\hline $25-29$ & 1109 & 18.6 \\
\hline $30-34$ & 725 & 12.2 \\
\hline $35-39$ & 689 & 11.6 \\
\hline $40-44$ & 515 & 8.6 \\
\hline $45-49$ & 439 & 7.4 \\
\hline \multicolumn{3}{|l|}{ Type of place of residence } \\
\hline Urban & 1636 & 27.4 \\
\hline Rural & 4327 & 72.6 \\
\hline \multicolumn{3}{|c|}{ Highest educational attainment } \\
\hline No education & 3636 & 61.0 \\
\hline Primary & 1327 & 22.3 \\
\hline Secondary & 861 & 14.4 \\
\hline Higher & 139 & 2.3 \\
\hline \multicolumn{3}{|l|}{ Marital status } \\
\hline Never married & 1553 & 26.0 \\
\hline Married/Living together & 3732 & 62.6 \\
\hline Widowed & 253 & 4.2 \\
\hline Divorced/Separated & 425 & 7.1 \\
\hline \multicolumn{3}{|l|}{ Region } \\
\hline SNNP & 1003 & 16.8 \\
\hline Oromiya & 971 & 16.3 \\
\hline Amhara & 827 & 13.9 \\
\hline Addis Abeba & 676 & 11.3 \\
\hline Tigray & 566 & 9.5 \\
\hline Benshagul Gumz & 398 & 6.7 \\
\hline Harari & 345 & 5.8 \\
\hline Gambela & 339 & 5.7 \\
\hline Dire Dawa & 298 & 5.0 \\
\hline Afar & 283 & 4.7 \\
\hline Somali & 257 & 4.3 \\
\hline \multicolumn{3}{|l|}{ Religion } \\
\hline Orthodox & 2858 & 47.9 \\
\hline Moslem & 1895 & 31.8 \\
\hline Protestant & 1023 & 17.2 \\
\hline Traditional & 72 & 1.2 \\
\hline Catholic & 68 & 1.1 \\
\hline Other & 47 & 0.8 \\
\hline \multicolumn{3}{|l|}{ Wealth index } \\
\hline Poorest & 1212 & 20.3 \\
\hline Poorer & 951 & 15.9 \\
\hline Middle & 931 & 15.6 \\
\hline Richer & 887 & 14.9 \\
\hline Richest & 1982 & 33.2 \\
\hline
\end{tabular}




\section{Prevalence of Anemia}

Among all respondents, the mean $( \pm s d)$ blood hemoglobin level (adjusted for altitude) was $12.7 \mathrm{~g} / \mathrm{dl}$ $( \pm 2.0 \mathrm{~g} / \mathrm{dl})$. Of the total, more than a quarter 1654 $(27.7 \%)$ were anemic. In this study, the region-wise composition of the study population was found to vary from what is reported in the recent Ethiopia census. Few regions were over-represented while others were underrepresented. Hence, the weighted prevalence (based on population size of each region) was computed as a correction. Accordingly, the weighted prevalence for any form of anemia was $27.4 \%$ (95\% CI: $26.3-28.5 \%$ ). The prevalence of mild, moderate and severe anemia were $17.8 \%, 8.2 \%$ and $1.4 \%$, respectively.

\section{Correlates of Anemia}

The mean hemoglobin level significantly varied across age categories. The highest level of $12.9 \mathrm{~g} / \mathrm{dl}$ was reported in the youngest (15-19 years) age group while the lowest level of $12.5 \mathrm{~g} / \mathrm{dl}$ in 35-39 years age group. The overall pattern showed an approximately linear decline between the ages of 15-39 years (Figure 1). Compared to the youngest group, the risk of anemia is significantly higher in the age group 30-34 and 35-39 years with adjusted odds ratio (AOR) of 1.31 (95\% CI: $1.07-1.60)$ and 1.56 (95\% CI: $1.27-1.90)$, respectively (Table 2).

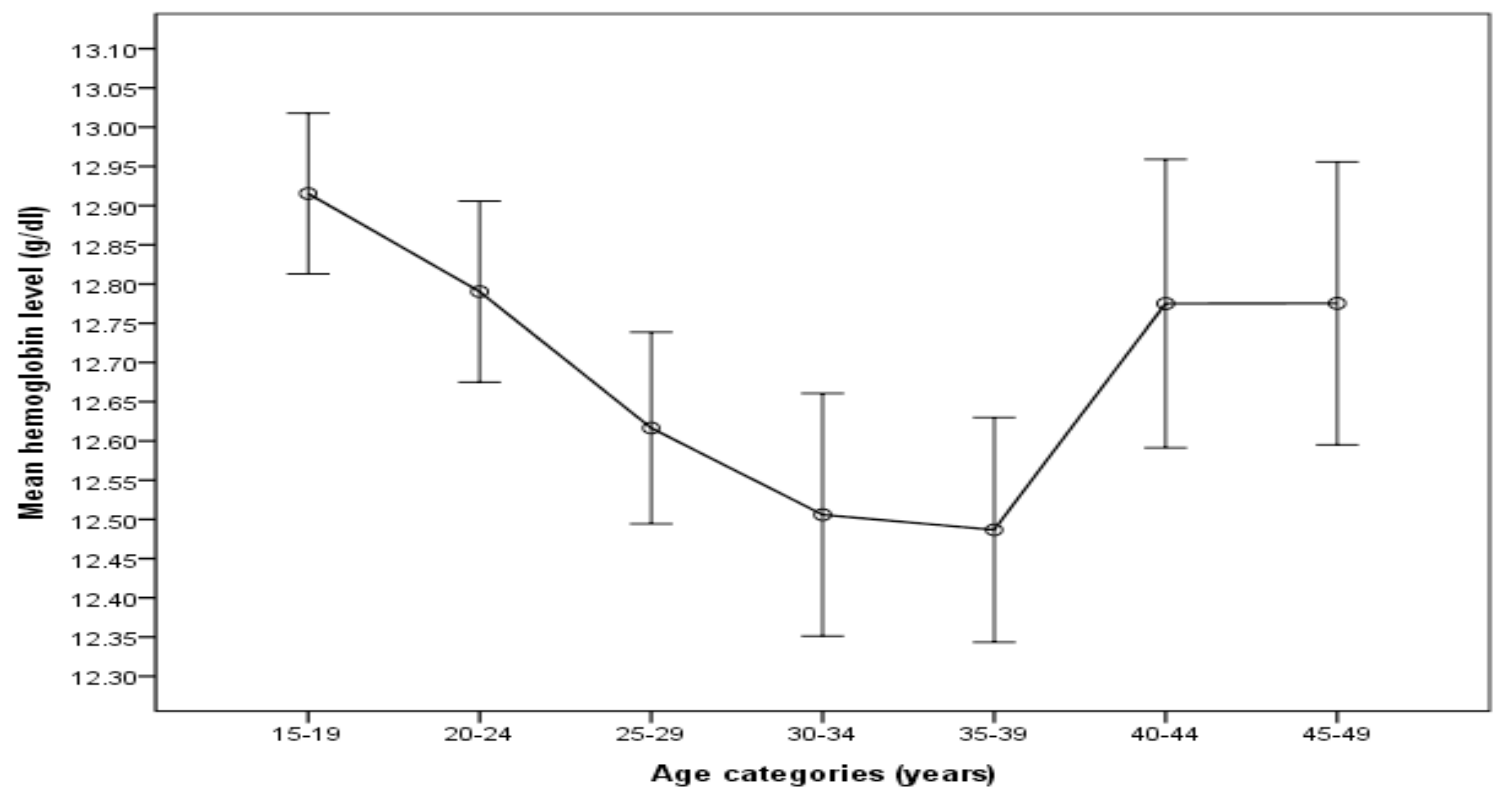

Figure 1: Mean blood hemoglobin level with $95 \% \mathrm{Cl}$ across different age categories, Ethiopia, 2005.

Religion, marital status and ethnicity of respondents did not have association with risk of anemia. Those women who were living in rural areas at the time of the survey were twice more likely to have anemia than urban dwellers with AOR of 1.99 (95\% CI: 1.73-2.30). Compared to women beyond secondary level of education, illiterates and those who had primary level education experienced significantly higher risk of anemia with AOR of 2.59 (95\% CI: 1.62-4.14) and $1.83(95 \%$ CI: 1.13-2.96), respectively. Likewise, the better the economic status of the women the lesser risk of anemia (Table 2).

Of 2527 mothers who gave at least one birth in the preceding 3 years of the survey, only $399(15.0 \%)$ consumed iron rich foods like meat, organ meat, fish and poultry in the reference period. The mean hemoglobin level among those who consumed iron rich foods (12.8 $\mathrm{g} / \mathrm{dl} \pm 1.9 \mathrm{~g} / \mathrm{dl})$ was significantly higher than those who didn't $(12.5 \mathrm{~g} / \mathrm{dl} \pm 2.1 \mathrm{~g} / \mathrm{dl})(p=0.019)$. The risk of anemia was 1.3 (95\%CI: 1.08-1.64) times higher among those who did not consume iron reach foods compared to their counterparts (Table 2).

The average $( \pm \mathrm{sd})$ dietary diversity score among mothers who had at least one delivery in the preceding 3 years of the survey was $4.01( \pm 1.53)$. The majority of the respondents $1241(49.5 \%)$ had low DDS. About 902 $(36.0 \%)$ and $364(14.5 \%)$ had medium and high DDS, respectively.

The mean DDS for pregnant and breastfeeding women were compared to other women in order to assess whether there is a better dietary diversity during these critical periods. Accordingly, the mean DDS for pregnant and non-pregnant women were found to be 3.84 and 4.03 , respectively. The DDS for pregnant women was significantly lower than for non-pregnant women $(p=0.032)$. The average scores for breastfeeding and nonbreastfeeding women were 3.99 and 4.08, respectively. The difference was not statistically significant. 
Table 2: Socio-demographic and nutritional variables as correlates of anemia among women of reproductive age, Ethiopia, 2005

\begin{tabular}{|c|c|c|c|c|}
\hline Variable & $\begin{array}{l}\text { Non } \\
\text { anemic }\end{array}$ & Anemic & COR $(95 \% \mathrm{CI})$ & AOR $(95 \% \mathrm{CI})^{\star *}$ \\
\hline Age & $(n=4309)$ & $(n=1654)$ & & \\
\hline $15-19$ & 1057 & 340 & 1 & 1 \\
\hline $20-24$ & 795 & 294 & $1.15(0.96-1.38)$ & $1.14(0.95-1.36)$ \\
\hline $25-29$ & 799 & 310 & $1.21(1.01-1.44)^{*}$ & $1.18(0.99-1.42)$ \\
\hline $30-34$ & 507 & 218 & $1.34(1.09-1.63)^{*}$ & $1.31(1.07-1.60)^{*}$ \\
\hline $35-39$ & 457 & 232 & $1.58(1.29-1.93)^{*}$ & $1.56(1.27-1.90)^{*}$ \\
\hline $40-44$ & 374 & 141 & $1.17(0.93-1.47)$ & $1.17(0.93-1.48)$ \\
\hline $45-49$ & 320 & 119 & $1.16(0.91-1.47)$ & $1.17(0.91-1.49)$ \\
\hline Place of residence & $(n=4309)$ & $(n=1654)$ & & \\
\hline Urban & 1335 & 301 & 1 & 1 \\
\hline Rural & 2974 & 1353 & $2.02(1.75-2.32)^{*}$ & $1.99(1.73-2.30)$ * \\
\hline Highest educational level & $(n=4309)$ & $(n=1654)$ & & \\
\hline No education & 2481 & 1155 & $2.62(1.64-4.18)$ * & $2.59(1.62-4.14)^{*}$ \\
\hline Primary & 1000 & 327 & $1.84(1.14-2.97)$ * & $1.83(1.13-2.96)^{*}$ \\
\hline Secondary & 710 & 151 & $1.20(0.73-1.96)$ * & $1.20(0.73-1.97)$ \\
\hline Higher & 118 & 21 & 1 & 1 \\
\hline Wealth index & $(n=4309)$ & $(n=1654)$ & & \\
\hline Poorest & 773 & 439 & $2.47(2.10-2.91)^{\star}$ & $2.45(2.08-2.88)^{*}$ \\
\hline Poorer & 635 & 316 & $2.17(1.82-2.59)^{*}$ & $2.15(1.80-2.56)^{*}$ \\
\hline Middle & 665 & 266 & $1.74(1.45-2.09)^{*}$ & $1.73(1.44-2.07)^{*}$ \\
\hline Richer & 624 & 263 & $1.84(1.53-2.21)^{*}$ & $1.82(1.52-2.19)^{*}$ \\
\hline Richest & 1612 & 370 & 1 & 1 \\
\hline Consumption of Iron rich foods & $(n=1736)$ & $(n=791)$ & & \\
\hline Yes & 288 & 111 & 1 & 1 \\
\hline No & 1448 & 680 & $1.22(0.96-1.55)$ & $1.34(1.08-1.64)^{*}$ \\
\hline Diet diversity level & $(n=1736)$ & $(n=791)$ & & \\
\hline Low & 1128 & 565 & $1.33(1.12-1.59)^{*}$ & $1.33(1.11-1.58)^{*}$ \\
\hline Medium or High & 608 & 226 & 1 & 1 \\
\hline BMI & $(n=3959)$ & $(n=1482)$ & & \\
\hline$<18.5$ & 973 & 466 & $1.36(1.19-1.56)^{*}$ & $1.36(1.19-1.56)^{*}$ \\
\hline $18.5-24.9$ & 2715 & 956 & 1 & 1 \\
\hline$\geq 25.0$ & 271 & 60 & $0.63(0.47-0.84)^{*}$ & $0.63(0.47-0.84)^{*}$ \\
\hline
\end{tabular}

** Adjusted for pregnancy status (except for BMI which was calculated among non-pregnant women).

* Significant association $(p<0.05)$.

COR Crude Odds Ratio.

AOR Adjusted odds Ratio.

The mean hemoglobin levels for low, medium and high diet diversity (DD) categories were $12.3 \mathrm{~g} / \mathrm{dl}( \pm 2.1 \mathrm{~g} / \mathrm{dl})$, $12.6 \mathrm{~g} / \mathrm{dl} \quad( \pm 1.8 \mathrm{~g} / \mathrm{dl}) \quad$ and $12.9 \quad \mathrm{~g} / \mathrm{dl} \quad( \pm 1.9 \mathrm{~g} / \mathrm{dl})$, respectively. One-way ANOVA indicated significant difference across the three categories $(P=0.000)$. Compared to those with medium or high DD level, the risk of anemia was 1.33 (95\% CI: 1.11-1.58) times higher among those with low DD level.

Among 5444 non-pregnant women whose body height and weight measurements were available, BMI was calculated as a measure of nutritional status. Accordingly, 1518 (27.9\%) had low BMI. The remaining $3595(66.0 \%)$ and $331(6.0 \%)$ had normal and above normal values. The mean hemoglobin levels for low, normal and overweight categories of BMI were $12.6 \mathrm{~g} / \mathrm{dl}$ $( \pm 2.1 \mathrm{~g} / \mathrm{dl}), 12.9 \mathrm{~g} / \mathrm{dl}( \pm 1.9 \mathrm{~g} / \mathrm{dl})$ and $13.3 \mathrm{~g} / \mathrm{dl}( \pm 1.9 \mathrm{~g} / \mathrm{dl})$, respectively. The difference was statistically significant $(P=0.000)$. Compared to those with normal BMI, women with low BMI were 1.36 (95\% CI: 1.19-1.56) times more likely to have anemia (Table 2).
About $474(7.9 \%)$ of all women were pregnant at the time of the survey. The hemoglobin level among pregnant women was $11.7 \mathrm{~g} / \mathrm{dl}( \pm 2.1 \mathrm{~g} / \mathrm{dl})$ and about $33.1 \%$ (95\%CI: $28.9-37.3 \%$ ) were anemic. Compared to non-pregnant, pregnant women had higher risk of anemia with OR of $1.32 \quad(95 \%$ CI: $1.08-1.61)$. The mean hemoglobin levels in the second and third trimesters were significantly lower than that of the corresponding value in the first trimester. Neither receiving ANC nor Ironfolate supplement during the pregnancy was associated with reduction in the risk of anemia (Table 3).

About one-third, 1962 (32.9\%) of respondents were breastfeeding at the time of the survey. The mean hemoglobin level among breastfeeding mothers was 12.6 $\mathrm{g} / \mathrm{dl}( \pm 2.0 \mathrm{~g} / \mathrm{dl})$ and $31.3 \%(95 \% \mathrm{CI}: 29.2-33.4 \%)$ of them were anemic. Compared to non-lactating mothers, lactating mothers had significantly higher risk of anemia with OR of 1.30 (95\% CI: 1.16-1.47). 


\begin{tabular}{|c|c|c|c|}
\hline Variable & Frequency & $\begin{array}{l}\text { Hemoglobin level }(\mathrm{g} / \mathrm{dl}) \\
\text { (Mean } \pm \text { SD) }\end{array}$ & $\begin{array}{l}\text { P value of ANOVA or } \\
\text { Independent t-test }\end{array}$ \\
\hline Gestational age & $(n=474)$ & & \\
\hline $1^{\text {st }}$ trimester & 97 & $12.2( \pm 2.3)$ & \\
\hline $2^{\text {nd }}$ trimester & 187 & $11.5( \pm 2.1)$ & $0.035^{*}$ \\
\hline $3^{\text {rd }}$ trimester & 190 & $11.5( \pm 1.8)$ & \\
\hline Received ANC & $(n=341)$ & & \\
\hline Yes & 91 & $11.6( \pm 2.2)$ & \\
\hline No & 250 & $11.6( \pm 1.8)$ & 0.946 \\
\hline Fe-Fol supplementation & $(n=340)$ & & \\
\hline Yes & 28 & $11.4( \pm 1.9)$ & \\
\hline No & 312 & $11.6( \pm 1.8)$ & 0.683 \\
\hline
\end{tabular}

The association between duration of breastfeeding and risk of anemia was assessed among 2247 mothers who had given one or more births in the preceding 3 years of the survey and had already ceased nursing. The mean hemoglobin levels for those who breastfed for less than 6 months, 6-11 months and 12 or more months were 12.3 $\mathrm{g} / \mathrm{dl}( \pm 2.4 \mathrm{~g} / \mathrm{dl}), 12.6 \mathrm{~g} / \mathrm{dl}( \pm 1.9 \mathrm{~g} / \mathrm{dl})$ and $12.5 \mathrm{~g} / \mathrm{dl}( \pm 2.0$ $\mathrm{g} / \mathrm{dl}$ ), respectively. The difference was not statistically significant.

With reference to respondents who had given no birth before, those with 1-2, 3-5 and more than 5 children had $1.47,1.51$ and 1.86 times higher risk of developing anemia. As the number of children born in the preceding 5 years of the survey increased, the risk of anemia also increased. Likewise, those women who gave birth in the month of the interview were 2.24 times more likely to develop anemia than their counterparts (Table 4).
Among 1362 women of reproductive age who gave two or more births in the preceding 5 years of the study, the effect of birth interval between the recent two births on risk anemia was assessed. However, no significant association was witnessed (Table 4).

Current use of contraceptives was associated with reduced risk of anemia. Those who were not using contraceptive were 1.36 times more likely to develop anemia than users. Among users, mean hemoglobin level did not vary significantly across contraceptive types (Table 4).

Among 988 mothers who delivered in the preceding 6 months of the survey, the effects of postnatal care, place of delivery and postpartum vitamin A supplementation history were assessed. However none of the variables were correlated with the risk of anemia (Table 4). 
Table 4: Potential reproductive health related correlates of anemia among women of reproductive age, Ethiopia, 2005

\begin{tabular}{|c|c|c|c|c|}
\hline Variable & Non anemic & Anemic & OR $(95 \% \mathrm{Cl})$ & AOR $(95 \% \mathrm{Cl})^{\star *}$ \\
\hline Children ever born (CEB) & $(n=4309)$ & $(n=1654)$ & & \\
\hline 0 & 1502 & 433 & 1 & 1 \\
\hline $1-2$ & 884 & 376 & $1.48(1.26-1.73)^{*}$ & $1.47(1.20-1.80)^{*}$ \\
\hline $3-5$ & 1034 & 427 & $1.43(1.23-1.73)^{*}$ & $1.51(1.19-1.91)^{*}$ \\
\hline$>5$ & 889 & 418 & $1.63(1.39-1.91)^{*}$ & $1.86(1.40-2.49)^{*}$ \\
\hline CEB in the preceding 5 years & $(n=4309)$ & $(n=1654)$ & & \\
\hline 0 & 2275 & 717 & 1 & 1 \\
\hline 1 & 1100 & 507 & $1.46(1.28-1.67)^{*}$ & $1.37(1.16-1.63)^{*}$ \\
\hline 2 & 802 & 355 & $1.40(1.21-1.63)^{*}$ & $1.31(1.06-1.63)^{*}$ \\
\hline 3 or more & 132 & 75 & $1.80(1.34-2.42)^{*}$ & $1.70(1.22-2.37)^{*}$ \\
\hline Gave birth in the month of the interview & $(n=4309)$ & $(n=1654)$ & & \\
\hline Yes & 4285 & 1634 & $2.19(1.20-4.00)^{*}$ & $2.24(1.23-4.07)^{*}$ \\
\hline No & 24 & 20 & 1 & 1 \\
\hline $\begin{array}{l}\text { Time interval between the recent two } \\
\text { births in the last } 5 \text { yrs }\end{array}$ & $(n=932)$ & $(n=430)$ & & \\
\hline $9-17$ months & 96 & 48 & $1.14(0.78-1.65)$ & $1.14(0.78-1.66)$ \\
\hline $18-23$ months & 158 & 84 & $1.21(0.90-1.63)$ & $1.21(0.90-1.61)$ \\
\hline 24 or more months & 678 & 298 & 1 & 1 \\
\hline Current contraceptive use & $(n=4309)$ & $(n=1654)$ & & \\
\hline No & 3743 & 1514 & $1.60(1.32-1.95)^{*}$ & $1.36(1.11-1.66)^{*}$ \\
\hline Yes & 566 & 140 & 1 & 1 \\
\hline Place of delivery & $(n=658)$ & $(n=330)$ & & \\
\hline Health institutions & 58 & 16 & 1 & 1 \\
\hline $\begin{array}{l}\text { Home } \\
\text { Received vitamin A supplementation in } \\
\text { the postpartum period after recent birth }\end{array}$ & $\begin{array}{c}600 \\
(n=658)\end{array}$ & $\begin{array}{c}314 \\
(n=330)\end{array}$ & $1.90(1.07-3.36)^{*}$ & $1.02(0.52-2.01)$ \\
\hline Yes & 140 & 61 & 1 & 1 \\
\hline No & 518 & 269 & $1.19(0.85-1.67)$ & $1.05(0.75-1.48)$ \\
\hline Had postnatal checkup & $(n=600)$ & $(n=314)$ & & \\
\hline Yes & 17 & 8 & 1 & 1 \\
\hline No & 583 & 306 & $1.15(0.48-2.61)$ & $0.91(0.38-2.20)$ \\
\hline
\end{tabular}

* Significant association $(p<0.05)$.

** Adjusted for place of residence, educational status, wealth index. COR Crude Odds Ratio.

AOR Adjusted Odds Ratio.

\section{Discussion}

More than a quarter of Ethiopian women of reproductive age were anemic. With reference to the WHO cutoff points (3), the magnitude indicates moderate public heath significance of anemia in Ethiopia. A recent study by Umeta et al (11) also documented a comparable prevalence of $30.4 \%$. Likewise, in 2006 MI Ethiopia estimated the prevalence as $27.0 \%$ (8). Among pregnant women about one third were anemic. This figure is higher than the 2002 estimate of UNICEF (17.4\%) (25) and what has been documented by Hayder et al (18.4\%) in 1999 (7). The findings over the decade did not witness tangible progress in the reduction of anemia in Ethiopia.

Women aged 25-39 years had the highest risk of anemia. This might be due to the fact that the age category is fertility intensive in women's life. According DHS Ethiopia 2005, approximately $60 \%$ of all births occurred in this age category (9). Studies conducted so far reported various patterns of association between age and of anemia. Studies in Ethiopia (10) and Tanzania (13) reported higher prevalence in older age groups. A study in Mexico documented higher prevalence in the 20-29 years of age than the younger or older age categories
(15); while, studies in Tanzania (18), Nigeria (14) and India (17) failed to witness any association.

Regarding other socio-demographic factors, being from lower economic and education category, and living in rural areas were identified as predisposing factors to anemia. Hence, empowering women in terms of education and economic status would have positive contributions to avert the problem.

The negative effect of women's reproductive role in causing anemia is clearly witnessed in this study. The risk of anemia was directly associated with cumulative fertility, fertility in the preceding five years of the survey and history of birth in the month of the interview. Similar studies in Ethiopia (7), Indonesia (26) and Mexico (15) also documented such association between parity and risk of anemia. The positive contribution of contraceptive use also provides supportive evidence in this regard.

Pregnancy is the most nutritionally demanding period in a woman's life. Consequently, pregnant women are advised to eat more diversified diet than usual. However, this wasn't the case in Ethiopia. The level of diet diversity among pregnant women was significantly lower 
than non-pregnant women. One possible explanation might be the presence of food taboos during pregnancy. According to a study in southern Ethiopia, 65\% of women avoided at least one food type during their recent pregnancy (27).

The role of breastfeeding as a predisposing factor for anemia is also overt in this study. Breastfeeding mothers were 1.3 times more likely to have anemia than nonbreastfeeding mothers. A study in Mexico reported a similar but stronger association (2.5 times higher risk) (15). Despite the scientific understanding that more diversified diet is needed during lactation, in this study the level of diet diversification among breastfeeding mothers was not better than non-breastfeeding mothers.

As pregnancy and delivery increase the risk of anemia, various components of maternity care are expected to mitigate this vulnerability. Providing of antenatal, postnatal care and skilled delivery attendance are believed to have beneficial contributions. However, this was not the case in the present study. Having one or more ANC, delivering at health institutions or having PNC did not contribute to the reduction of risk of anima.

Similarly, among pregnant women taking iron-folate supplement did not contribute to lessening the risk of anemia. However, this is not consistent with the scientific understanding that the supplement is effective in improving the hematologic indices of pregnant women (28-30). This unexpected finding might be due noncompliance of study subjects to regimen of the supplementation. According to DHS 2005, only $11 \%$ of Ethiopian pregnant women took iron-folate supplement during their recent pregnancy and nearly all of them took the supplement for not more than 60 days (9).

Vitamin A deficiency can contribute to anemia as retinol and its metabolites are involved in the formation of erythrocytes and mobilization of stored iron (21). However, such a finding was not witnessed in this study. Among women who gave birth in the preceding 6 months of the survey, postpartum vitamin A supplementation was not associated with the risk of anemia.

\section{Conclusion and Recommendation}

Anemia has moderate public health significance in Ethiopia. The overall level of diet diversity and consumption of iron rich foods were enormously low. DDS was even lower among pregnant women. Living in rural areas, being from the lower economic and educational status categories were important predisposing factors to anemia. Breastfeeding and high parity increases risk of anemia significantly; whereas, contraceptive use reduces the risk. Utilization of maternity services, taking iron-folate supplement during pregnancy and vitamin A supplement in the postpartum period did not significantly associate with the reduction of the risk of anemia.
Family planning contributes crucially in reducing the burden of anemia in women of reproductive age. Economic and educational empowerments of women have affirmative inputs in this regard. A combination of nutrition education and livelihood promotion strategies should be instated to enhance diet diversity especially during pregnancy and breastfeeding. Maternal nutrition interventions should be integrated into maternity services. The compliance of Ethiopian mothers to prenatal iron supplement should be assessed.

\section{Acknowledgment}

The authors acknowledge MEASURE for granting access to Ethiopian DHS 2005 data.

\section{References}

1. McLean E, Cogswell M, Egli I, Wojdyla D, Benoist B. Worldwide Prevalence of Anemia: WHO Vitamin and Mineral Nutrition Information System 19932005. Public Health Nutr 2008;12(4):444-54.

2. World Health Organization. The Prevalence of Anemia in Women: A Tabulation of Available Information, $2^{\text {nd }}$ ed., 1992.

3. World Health Organization, Centers for Disease Control and Prevention. Worldwide Prevalence of Anemia: WHO Global Database of Anemia, 2008.

4. Nowrousian MR. Recombinant Human Erythropoietin in Clinical Oncology, $2^{\text {nd }} e d$., New York: Springer, 2008.

5. Parul Christian. Iron Deficiency and Anemia: Causes, Consequences and Solutions [document on the internet]. John Hopkins Bloomberg School of Pubic Health; 2005 [Cited 2009 Dec 31]. Available from: http://ocw.jhsph.edu/courses/International Nutrition/PDFs/Lecture5.pdf.

6. World Health Organization. The World Health Report 2002: Reducing Risk and Promoting Healthy Life [document on the internet]. The institute; [Cited 2009 Oct 20]. Available from: http://www.who.int/whr/2002/en/.

7. Haidar J, Nekatibeb H, Urga K. Iron Deficiency Anemia in Pregnant and Lactating Mothers in Rural Ethiopia. East Afri Med J 1999;76(11):618-22.

8. Micronutrient Initiative. Ethiopian Country Profile [document on the internet]. The institute 2006; [Cited 2009 Dec 20]. Available from: http://www.micronutrient.org.

9. ORC Macro and CSA. Ethiopian Demographic and Health Survey 2005, 2006.

10. Haidar J, Pobocik R. Iron Deficiency Anemia is not a rare problem among women of reproductive ages in Ethiopia: A Community based cross-sectional study. BMC Blood Disorders 2009;9(7).

11. Umeta M, Haidar J, Demissie T, Akalu G, Ayana G. Iron Deficiency Anemia among Women of Reproductive Age in Nine Administrative Regions of Ethiopia. Ethiop J Health Dev 2008;22(3):252-8.

12. Hwalla N, Adra N. Iron Deficiency is an Important Contributor to Anemia among Reproductive Age 
Women in Lebanon. Ecology of Food and Nutr 2004;43:77-92.

13. Hinderaker SG, Olsen BE, Bergsjø P, Lie RT, Gasheka P, Kvåle G. Anemia in Pregnancy in the Highlands of Tanzania. Acta Obstet Gynecol Scand 2001;80:18-26.

14. Dim CC, Onah HE. The Prevalence of Anemia among Pregnant Women at Booking in Enugu, Southeastern Nigeria. Med Gen Med 2007;9(3):11.

15. Monárrez-Espino J, Martínez $H$. Iron Deficiency Anemia in Tarahumara Women of Reproductive Age in Northern Mexico. Salud Pública Méx 2001;43(5):1-5.

16. Antelman G, Msamanga GI, Spiegelman D, Urassa EJ, Narh R, Hunter DJ, et al. Nutritional Factors and Infectious Disease Contribute to Anemia in Pregnant Women with HIV in Tanzania. J Nutr 2000; 130: 1950-7.

17. Bentley ME, Griffiths PL. The Burden of Anemia among Women in India. Eur $J$ Clin Nutr 2003;57:52-60.

18. Massawe SN, Urassa EN, Nystrom L, Lindmark G. Anemia in Women of Reproductive Age in Dar-essalaam, Tanzania. East Afri Med J 2002;79(9):4616.

19. Federal Democratic Republic of Ethiopia-Population Census Commission. Summary and Statistical Reports of the 2007 Population and Housing Census: Population Size by Age and Sex, Dec 2008.

20. Ministry of Health-Ethiopia. Country Profile [document on the internet]. The institute 2009; [Cited 2009 Dec 26]. Available from: http://www.moh.gov.et/index.php?option=com.

21. Swindale A, Bilinsky P. Household Dietary Diversity Score for Measurement of Household Food Access: Indicator Guide Version 2, Washington, D.C, Food and Nutrition Technical Assistance Project, Academy for Educational Development, 2006.

22. Food and Agriculture Organization of the United Nations. Guidelines for Measuring Household and Individual Dietary Diversity: Version 3, FAO, Rome, 2007.
23. Food and Agriculture Organization of the United Nations. Baseline Survey Report Protecting and Improving Household Food Security and Nutrition in HIV/AIDS Affected Areas in Manica and Sofala Province, Maputo, Mozambique, 2006.

24. Savy M, Martin-Pre'vel Y, Traissac P, Delpeuch F. Measuring Dietary Diversity in Rural Burkina Faso: Comparison of a 1-day and a 3-day Dietary Recall. Public Health Nutr 2007;10(1):71-8.

25. United Nations Development Pprogram Emergency Unit for Ethiopia. Primary health Care in Ethiopia [document on the internet]. University of Pennsylvania; 2000 [Cited 2009 Feb 13]. Available from: http://www.africa.upenn.edu/eue_web/eue_mnu.htm.

26. Prihatini S. Determinant Factors of anemia in Women of Reproductive Age in Two Provinces in Indonesia. Penel Eating Nutr 2008;31(1):8-14.

27. Tsegaye D. Food Aversions and Cravings during Pregnancy: Prevalence and Significance for Maternal Nutrition in Ethiopia. Food Nutr Bull 1998;19(1):1-7.

28. Agarwal T, Kochar GK, Goel S. Impact of Iron Supplementation on Anemia during Pregnancy. Ethno Med 2008;2(2):149-151.

29. Trugo MF, Donangelo CM, Seyfarth SP, Henriques C, Andrade LP. Folate and Iron Status of Nonanemic Women during Pregnancy: Effect of Routine Folate and Iron Supplementation and Relation of Erythrocyte Folate with Iron Stores. Nutr Res 1996;16 (8):1267-76.

30. Feightner JW. Routine Iron Supplementation during Pregnancy [document on the internet]. Public Health Agency of Canada 2009; [Cited 2009 Dec 23]. Available from: http://www.phac-aspc.gc.ca/ publicat/clinic-clinique/pdf/s1c6e.pdf.

31. World Health Organization, Food and Agriculture Organization of the United Nations. Vitamin and Mineral Requirement in Human Nutrition, $2^{\text {nd }} e d$., Bangkok: WHO press, 1998. 